\title{
Moving the Needle on Atherosclerotic Cardiovascular Disease and Heart Failure with Influenza Vaccination
}

\author{
Bahar Behrouzi ${ }^{1,2,3,4}$ (D) Jacob A. Udell ${ }^{1,2,3,4,5}$ (D) \\ Accepted: 2 August 2021 / Published online: 21 October 2021 \\ (C) The Author(s), under exclusive licence to Springer Science+Business Media, LLC, part of Springer Nature 2021
}

\begin{abstract}
Purpose of Review The interplay between viral respiratory infections and cardiovascular disease has been most comprehensively researched using seasonal and pandemic influenza viruses as case studies. Here, we summarize the latest international observational research and clinical trials that examined the association between influenza, influenza vaccines, and cardiovascular disease, while contextualizing their findings within those of landmark studies.

Recent Findings Most recent observational literature found that one in eight adults hospitalized with laboratory-confirmed influenza infection experienced an acute cardiovascular event. The latest meta-analysis of the cardioprotective effects of influenza vaccine found a $25 \%$ reduced risk of all-cause death. There are four large cardiovascular outcome trials assessing the cardioprotective effects of different influenza vaccine strategies. Among these, the INVESTED study showed there is no significant difference between the high-dose trivalent and standard-dose quadrivalent influenza vaccines in reducing allcause mortality or cardiopulmonary hospitalizations in a high-risk patient group with pre-existing cardiovascular disease. Summary Persons with cardiovascular disease represent a high priority group for viral vaccines; hence, using robust evidence to increase vaccine confidence among patients and practitioners is integral as we prepare for a possible influenza resurgence in the coming years.
\end{abstract}

Keywords Influenza vaccines $\cdot$ Universal vaccines $\cdot$ ASCVD $\cdot$ Pandemics $\cdot$ Vaccine hesitancy $\cdot$ Post-viral syndromes

This article is part of the Topical Collection on Cardiovascular Disease and Stroke

Jacob A. Udell

jay.udell@utoronto.ca

Bahar Behrouzi

bahar.behrouzihoma@mail.utoronto.ca

1 ICES, Toronto, ON, Canada

2 Cardiovascular Division, Department of Medicine, Women's College Hospital, 76 Grenville Street, Toronto, ON M5S 1B2, Canada

3 Institute of Health Policy, Management, and Evaluation, University of Toronto, Toronto, ON, Canada

4 Temerty Faculty of Medicine, University of Toronto, Toronto, ON, Canada

5 Peter Munk Cardiac Centre, University Health Network, Toronto, ON, Canada

\section{Introduction: Vicious Cycle of Viral Respiratory Infections and Cardiovascular Disease}

Since 2015, heart disease has been the leading cause of death in the United States (US), with a 5\% increase from 2019 to 2020 alone — the largest jump since 2012 [1]. Influenza and pneumonia have also prominently featured among the top ten causes of deaths since 2015, with an increase in deaths by $7.5 \%$ from 2019 to 2020 [1,2]. Now, because of the Coronavirus Disease 2019 (COVID-19) pandemic, it has never been more apparent to the public that there exists a vicious cycle between viral respiratory infections (VRI) and cardiovascular disease (CVD). Underlying CVD puts patients at increased risk of VRI and downstream cardiopulmonary sequelae, such as those due to SARS-CoV-2 [3, 4], influenza viruses $[5,6,7 \bullet \bullet]$, and respiratory syncytial virus [8-10]. Reciprocally, VRI can create a systemic inflammatory environment that is conducive to major adverse cardiovascular (CV) events [10, 11, 12•, 13-16]. Yet, this 
vicious cycle is not a new concept. One of the earliest published reports of an association between respiratory infection and higher $\mathrm{CV}$ mortality referred to the 1918 influenza pandemic, which was caused by influenza A (H1N1) virus. During the influenza epidemics of 1918-1919 and 1920, $18 \%$ of excess deaths were attributed to "organic heart diseases." Subsequently, during the 1922, 1923, 1926, and 1928-1929 epidemics, the attribution increased to $46 \%$ of excess deaths [17]. Since then, seasonal influenza epidemics have been, and continue to be, associated with populationlevel increases in CV hospitalizations and mortality.

Prior to the COVID-19 pandemic, in the US alone, influenza infections were associated with 225,000 hospitalizations, 36,000 cardiopulmonary deaths, and 51,000 deaths in a typical year [18, 19]. Furthermore, approximately half of adults who were hospitalized with influenza infection had CVD [20]. During the 2019-2020 influenza season, the US Centers for Disease Control and Prevention (CDC) estimated 38 million influenza illnesses (equivalent to the population of California), 400,000 influenza hospitalizations (about the population of Miami, FL.), and 22,000 influenza deaths, which is enough people to fill Madison Square Garden in New York City [21]. This burden, in turn, continued to disproportionately impact the most vulnerable in a population, such as people experiencing multimorbidity and/or immune compromise and older adults, the latter accounting for $43 \%$ of hospitalizations and $62 \%$ of deaths [21-23]. Although the 2019-2020 season was described to be of moderate severity, it was especially severe for children under 4 years old and working age adults (18 to 49 years old), for whom these rates of infection, morbidity, and mortality were higher than those observed in the 2017-2018 season, i.e., a recent season with high severity (Fig. 1), and with hospitalization rates higher than those observed during the $2009 \mathrm{H} 1 \mathrm{~N} 1$ pandemic [24, 25].

\section{The Latest Studies Investigating Influenza and CVD}

The landmark studies that highlighted this association over the past two decades have been discussed in-depth elsewhere [12•]. However, in the past year, additional large and welldesigned studies have emerged that add further credence to the growing interest and literature base interrogating this disease intersection. In a recent cross-sectional study of 80,261 adults from the US Influenza Hospitalization Surveillance Network (FluSurv-NET; covering 9\% of the US population) across 8 influenza seasons (2010-2018), the CDC found that almost one in eight adult patients hospitalized with laboratory-confirmed influenza infection experienced an acute CV event $[7 \bullet \bullet]$. The most common were heart failure $(6.2 \%)$ and ischemic heart disease (5.7\%), whereas less common CV event types included hypertensive crisis (1.0\%), cardiogenic shock $(0.3 \%)$, acute myocarditis $(0.1 \%)$, acute pericarditis $(0.05 \%)$, and cardiac tamponade $(0.02 \%)$. The high incidence of acute congestive heart failure (HF) is especially notable in the context of the very low incidence of acute viral myo-/pericarditis and given approximately half (49.2\%) of patients with HF had no prior diagnosis of HF nor cardiomyopathy [26]. The findings for ischemic heart disease also point to the need for further investigation of ischemic stroke associated with influenza, given the shared pathogenic and cardioembolic mechanisms. Due to limitations in discerning the acuity of conditions based on International Classification of Diseases (ICD) discharge codes alone, the investigators excluded diagnoses for cardiac arrhythmias, including atrial fibrillation, although association with this $\mathrm{CV}$ event type and mitigation with influenza vaccination has been reported [27, 28].

Naturally, these same patients merit higher care needs and demonstrate higher healthcare utilization as captured by traditional metrics. In the above study, patients experiencing acute $\mathrm{CV}$ events had a median length of stay of 5 days, with a third requiring intensive care and $7.3 \%$ dying in hospital. Of those who died, $25 \%$ had an associated acute $\mathrm{CV}$ event, which may have been preventable had the initial influenza infection been averted. Additionally, as has been seen repeatedly in similar studies, most of these patients were already experiencing multimorbidity at baseline, with events having occurred in $20.6 \%$ of hospitalized patients with chronic CVD, $19.3 \%$ of those with chronic renal disease, and $14.8 \%$ of those with diabetes. Predictors of ischemic heart disease or acute HF were older age, extreme obesity (body mass index $\geq 40.0 \mathrm{~kg} / \mathrm{m}^{2}$ ), current tobacco use, chronic HF or cardiomyopathy, coronary artery disease (CAD), diabetes, and chronic renal disease. Atrial fibrillation was additionally found to be a predictor of acute HF.

Although the gold standard method for ascertaining positive influenza infection is using laboratory confirmation, in practice, most patients experiencing influenza infections are not formally diagnosed this way. Additionally, in the US, it has been previously shown that influenza-related hospitalizations are likely under-detected by more than threefold for adults aged 18 to 65 years, and by more than fivefold for older adults [29]. Hence, many of the best retrospective analyses discussed here and previously [12•] that examine the association between laboratory-confirmed influenza infection and CV sequelae are likely underestimating the true burden of influenza infection and associated CV morbidity and mortality.

Further, influenza virus circulation patterns and low temperatures are closely related in temperate zones, which have more distinct seasonal changes compared to tropical climates. Given environmental factors common during the winter season such as low temperature and humidity may 


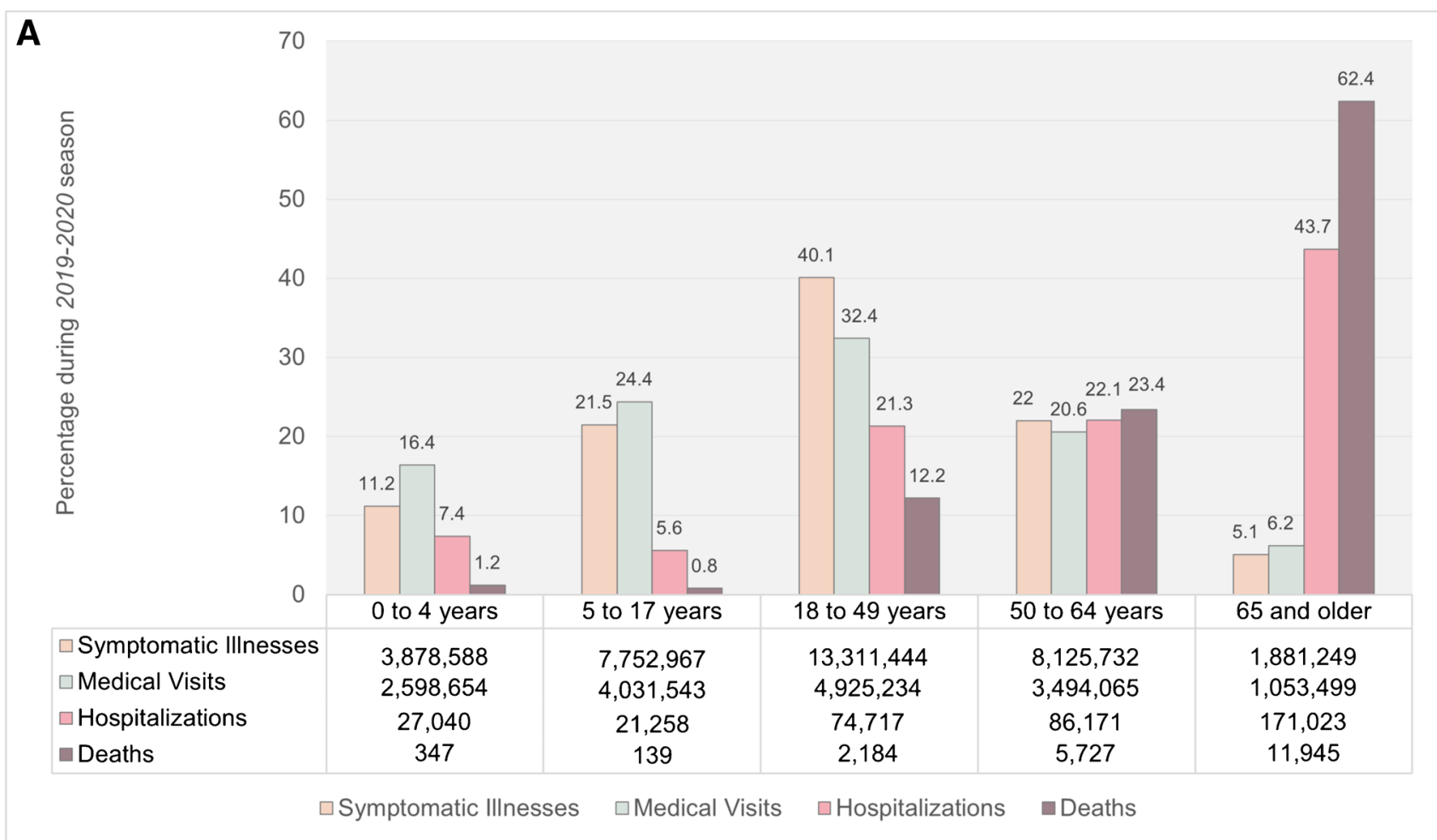

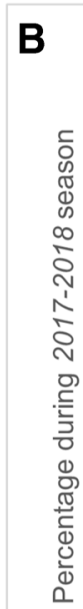

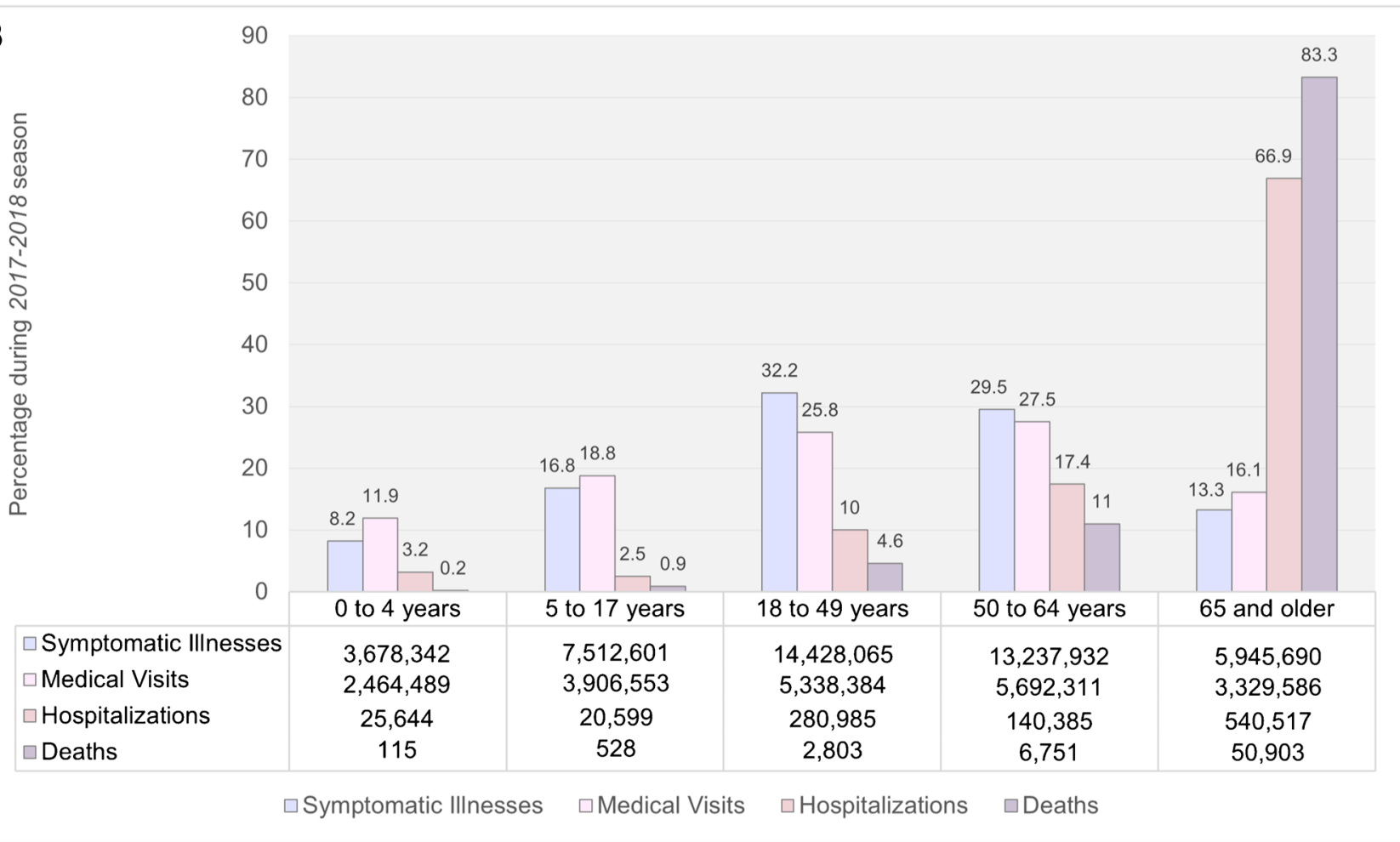

Fig. 1 Estimated percentage of influenza-related illnesses, medical visits, hospitalizations, and deaths by age group for the (A) moderate severity 2019-2020 influenza season versus the (B) high severity
2017-2018 influenza season (data from the CDC) [21]. The absolute numbers of events are shown in the data table below each graph. 
predispose persons to prothrombotic states, it has been questioned whether the association between influenza infection and adverse $\mathrm{CV}$ outcomes is confounded by climate factors $[30,31]$. For instance, excess winter mortality and acute myocardial infarction (MI) risk has been attributed to low temperatures [30, 31]. However, recent observational studies in the US, England, Scotland, Denmark, and Spain, predominantly employing time-series analyses or self-controlled case series analyses, have shown that events like HF, stroke, and MI-associated hospitalizations and deaths are likely attributable to influenza epidemics [32-36, 37••]. Time-series and self-controlled case series analyses are elegant observational design and analytical methods developed to mitigate the challenges of investigating causal associations that account for confounders and better isolate the effect of influenza on cause-specific events. However, the interpretation of these population-level studies is not immediately intuitive, and the magnitude of reported effects has been variable.

In the latest time-series analysis, García-Lledó and colleagues analyzed the temporal relationship between influenza-like illness incidence and type 1 acute MI, while adjusting for ambient minimum temperature, over five influenza seasons (2013 to 2018) in Madrid, Spain [37••]. This is the first study using a time-series analysis (with quasiPoisson regression and distributed lag-nonlinear models) to make a distinction between type 1 and type 2 MI, with 8240 cases of type 1 identified via angiography and recorded in a regional primary angioplasty database. Adjusting for year, month, and temperature during influenza seasons, they found a relative risk (RR) of 1.23 (95\% CI, 1.03-1.47), which was consistent across sexes and age groups, although marginally non-significant for males and younger persons. The lag-nonlinear models showed that an increase of influenza rates by 50 cases per 100,000 in a week resulted in a $16 \%$ increased RR of type 1 acute MI (95\% CI, 1.09-1.23) during the same week but disappeared 1 week after (RR, 0.95; 95\% CI, 0.89-1.05). At the same time, there was a linearly increased risk of $2.5 \%$ per $1{ }^{\circ} \mathrm{C}$ decrease in the minimum temperature, showing a smaller and independent effect of temperature on type 1 acute MI after adjusting for influenza.

\section{Cardioprotective Effects of Influenza Vaccines}

In the above CDC-FluSurv-NET study, less than half (47.2\%) of the study population had received an influenza vaccine, with $39.2 \%$ not having received it and $13.6 \%$ with unknown vaccination status $[7 \bullet \bullet]$. This is unfortunate given that people vaccinated at least 2 weeks before hospitalization had a lower risk of HF (adjusted RR 0.86; 95\% CI, $0.80-0.92$ ) and ischemic heart disease (adjusted RR 0.80; 95\% CI, 0.74-0.87) when compared with those who did not get a vaccine $[7 \bullet \bullet$. Similarly, the recent work by GarcíaLledó and colleagues also found that for patients above 59 years of age, influenza vaccination was associated with a decreased risk of type 1 acute MI (60 to 64 years: RR 0.58 ; $95 \%$ CI, $0.47-0.71$ and $\geq 65$ years: RR 0.53 ; 95\% CI, $0.49-0.57)[37 \bullet \bullet]$.

Prior to these studies, several large meta-analyses of observational and trial data have shown that influenza vaccination is associated with a reduction in mortality and other adverse outcomes, including those of $\mathrm{CV}$ etiology, in adults with influenza infection [38-40]. A recent meta-analysis found that influenza vaccination was associated with (i) a $25 \%$ reduced risk of all-cause death and (ii) an 18\% (based on observational data) or 56\% (based on randomized controlled trial data) reduced risk of CV mortality [41]. These effect sizes are comparable to guideline-directed therapy with $\beta$-blockers and angiotensin-converting enzyme inhibitors, which have shown mortality reductions from $\approx 20$ to $25 \%$ [42-45]. In this context, the well-established safety, accessibility, feasibility, and cost-effectiveness of influenza vaccination make it a compelling first-line intervention in the armamentarium of CV prevention therapies [46].

However, many high-risk patients experiencing multimorbidity, including those with CVD, have an altered immune response to influenza vaccine as a result of processes like immunosenescence and inflammaging [47, 48], potentially resulting in reduced effectiveness for preventing clinical events [49]. Even at baseline, influenza vaccine effectiveness among the general populations has been waning in recent years, due to antigenic drift and shift, as well as egg adaptation [50-52]. As a result, newer and stronger vaccine formulations, in terms of dose and coverage of viral strains, are being developed while the technology for a universal influenza vaccine is an aspirational goal to meet contemporary needs $[53,54]$.

One such example is a high-dose seasonal influenza vaccine, containing four times the amount of hemagglutinin antigen than standard-dose vaccines and typically used in older adults with relatively weaker immune systems, which has been shown to augment the immune response in cardiac patients. In a Phase IIIb-IV, randomized, active-controlled trial against standard-dose trivalent influenza vaccine over two influenza seasons (2011-2012 and 2012-2013), the high-dose trivalent vaccine induced significantly higher antibody responses and provided better protection against laboratory-confirmed, symptomatic influenza illness (relative efficacy, 24.2\%; 95\% CI, 9.7-36.5) among 31,989 participants aged 65 and up from 126 research centers in the US and Canada [55]. Around the same time, Van Ermen and colleagues randomized 28 patients with heart failure to double-dose versus standard-dose influenza vaccine and found that antibody production, assessed as log haemagglutination unit changes, were significantly higher at 2 to 4 weeks 
following the higher dose vaccine (3.3 vs. 1.6 for $\mathrm{A} / \mathrm{H} 3 \mathrm{~N} 2$, $p<0.001 ; 1.9$ and 1.1 for $\mathrm{A} / \mathrm{H} 1 \mathrm{~N} 1, p=0.009$; and 1.7 and 1 for B-type, $p=0.02$ ) [56]. Together, this evidence suggests a high-dose influenza vaccination strategy may further reduce morbidity and mortality, thus better serving as a secondary prevention measure [57].

So far, the Influenza Vaccine to Effectively Stop Cardio Thoracic Events and Decompensated heart failure (INVESTED) trial is the largest ( $n=5260$ participants) and longest (3 influenza seasons) cardiovascular outcome trial (CVOT) to have assessed the comparative effectiveness of a high-dose trivalent influenza vaccine versus a standard-dose quadrivalent vaccine in reducing cardiopulmonary events in a high-risk $\mathrm{CV}$ population [58]. The investigators did not find a significant difference between the strategies in reducing all-cause mortality or cardiopulmonary hospitalizations in this high-risk group. The possible reasons for this are multifactorial, notwithstanding the wider strain coverage conferred by the standard-dose quadrivalent vaccine $[59,60 \bullet \bullet$.

Now, given a quadrivalent formulation of the high-dose vaccine has emerged, containing an additional influenza $B$ strain lineage, it is the next candidate for evaluation via a CVOT in the improvement of protection against influenza and associated complications in high-risk patient groups (NCT04137887) [61••]. This ongoing study by Hollingsworth and colleagues combines the gold standard method of a Phase III/IV, modified double-blinded, randomized controlled trial with real-world pragmatic data collection through national registries. They aim to assess the relative effectiveness of the high-dose versus the standard-dose quadrivalent influenza vaccine in preventing cardiopulmonary hospitalizations up to 6 months post-vaccination, in $\geq 120,000$ adults aged 65 and up across Finland, over multiple influenza seasons.

Aside from INVESTED and NCT04137887, there are two other ongoing CVOTs (Fig. 2), the characteristics of which have been summarized elsewhere [12•, 62•, 63•]. Briefly, the Influenza Vaccination After Myocardial Infarction (IAMI) pragmatic, registry-based trial is randomizing patients with acute coronary syndrome undergoing coronary angiography to the standard-dose influenza vaccine versus placebo in 8 countries across Asia, Australia, and Europe [62•]. The Influenza Vaccine in Patients With Heart Failure to Reduce Adverse Vascular Events (IVVE) trial is randomizing patients with heart failure to the standard-dose influenza vaccine versus placebo in 10 countries across Asia, the Middle East, and Africa [63•]. Notably, all these CVOTs are specifically including patients with pre-existing CVD of varying manifestations, except for NCT04137887, which is mainly assessing older adults. Further, both INVESTED and NCT04137887 are active-controlled trials, with the standard-dose quadrivalent vaccine administered to participants in the comparator arm. This was to maintain equipoise since the vaccine was projected to become (or already is) the standard of care in those trial regions, instantiated by clear efficacy data and strong recommendations supporting vaccination in patients with CVD [64]. IAMI and IVVE, however, are placebo-controlled trials for varying reasons. IAMI's target patient population included hospitalized patients, who are not typically vaccinated unless out of hospital, and was enriched for participants who are specifically not considering vaccination. Further, annual influenza vaccination coverage for Scandinavian countries is approximately $50 \%$ [65]. As a result, the trial was effectively increasing

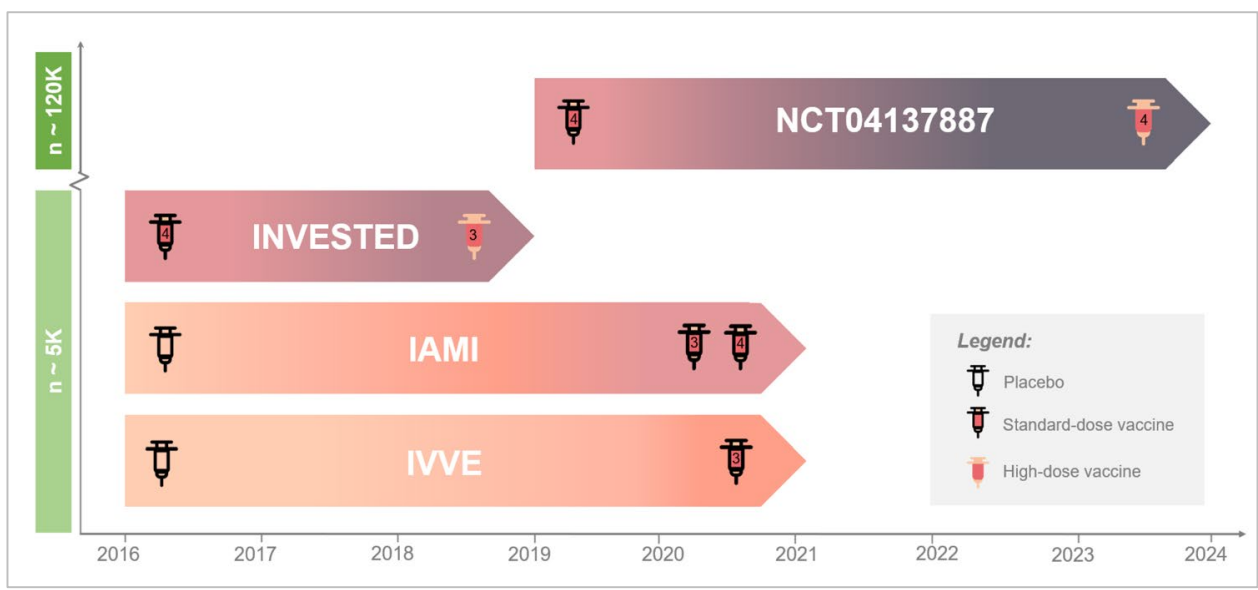

Fig. 2 Summary schematic of the four global influenza vaccine cardiovascular outcome trials, with study years indicated on the horizontal axis and approximate sample size indicated on the vertical axis $[60 \bullet \bullet, 61 \bullet \bullet, 62 \bullet, 63 \bullet]$. The number within each vaccine icon indicates the valence used within each two-arm trial. The influenza vaccine intervention of interest is shown to the right of each trial name, whereas the comparator/control intervention is shown to the left. Note that IAMI, as a pragmatic trial, switched partway from assessing the standard-dose trivalent to the standard-dose quadrivalent influenza vaccine as the main intervention, given it became the standard of care. Abbreviations: $K$, thousand participants; $n$, sample size; 3 , trivalent; 4, quadrivalent; , approximately 
vaccination coverage among their eligible participants. In contrast, IVVE permitted participants to receive influenza vaccine outside of the trial if they chose to do so or if prescribed, given there is no evidence of harm from receiving two influenza vaccinations. But, like IAMI, they also tried to enrich for participants who are not routinely vaccinated against influenza by excluding those who had received the vaccine in two of the three prior years. As a result, this trial was also increasing vaccination coverage among their participants, in practice.

Overall, patients with or at risk of CVD represent an important target population for the rapidly developing field of viral vaccines. Further, knowledge gained from these patients is applicable to most other chronic medical conditions that are susceptible to the same underlying pathophysiologic immune-modulation and frailty mechanisms [66]. This is especially important in the context of the current COVID-19 pandemic, which has exposed the interrelatedness of our societies in accelerating disease spread and its implications for future pandemics. The reduced global circulation of influenza virus throughout 2020 and early 2021 may increase the number of people with reduced immunity to influenza through lack of exposure [67, 68]. Following the widespread administration of SARS-CoV-2 vaccines, resumptions in international travel, and relaxation of nonpharmaceutical public health measures, it is inevitable that influenza will return [69]. Aside from returning as a seasonal virus, with potential for some lineages disappearing while new quasi-species or drifted variants emerge over time, influenza also continues to present a pandemic threat [67]. Hence, a clear, global, public health strategy supporting influenza vaccination among all eligible individuals over 6 months of age carries even greater importance.

\section{Conclusion - a Shot at the Future: Continuing Surveillance and Addressing Vaccine Hesitancy}

Even with the available evidence, influenza immunization rates remain low among the general population and highrisk individuals [70]. For example, in a recent US study of 19,793 patients with atherosclerotic CVD, 32.7\% lacked influenza vaccination, despite guideline recommendations [71-73]. Significant socioeconomic and demographic disparities also exist in vaccination coverage among high-risk CV patients [74]. Vaccine hesitancy was identified in 2019 by the World Health Organization as one of the ten major threats to global health and has been exacerbated by the COVID-19 pandemic, exposing global chasms in equitable vaccine access and deployment [75]. After 15 months and counting of global morbidity, mortality, detrimental economic, and social effects, and despite strict non-pharmaceutical interventions and lightning-fast development of effective viral vaccines, a lack of vaccine confidence still persists [76].

Many of the studies described here often concluded with calls for general practitioners and specialists to actively participate in vaccine counseling with their patients. We propose that framing this recommendation within the context of the three Cs framework for addressing vaccine hesitancy (complacency, convenience, and confidence) will maximize its impact [77]. A recent study suggests that vaccine uptake among older adults can be boosted by emphasizing the protective effects of influenza vaccination against increased risk of ischemic heart disease and stroke in a short and focused encounter with a physician [78]. This was shown to motivate at least $51 \%$ of their patient sample, who had previously declined vaccination. This serves as an example of boosting confidence in the effectiveness of influenza vaccines while also mitigating $\mathrm{com}$ placency in that the patient's focus is also directed to postviral complications like "heart attack and stroke," which conventionally carry more vivid risks than influenza-like illnesses. Bhugra and colleagues have also newly summarized key strategies in the literature for ameliorating influenza vaccine uptake among patients with CVD, through improved understanding of the key social determinants of health and vaccine-related behaviors, with applications for increasing vaccine coverage for other viral vaccines [79•]. Reviews that highlight the pros and cons of study design and analytical features and concisely explain the benefits and safety of influenza vaccine are designed to inform practitioners and boost confidence in study results. Consequently, practitioner confidence can improve advocacy to increase vaccine confidence among healthcare workers as well. Addressing convenience will require larger coordinated responses between global public health bodies and vaccine manufacturers to dismantle the structural hurdles to improve equitable vaccine access but in the interim can be initiated at the grassroots level via the therapeutic relationships that exist between healthcare workers and their patients.

In addition, underscoring the CV sequelae following influenza infection draws natural parallels to the post-acute sequelae currently emerging for COVID-19, i.e., "long covid" [80]. In fact, a variety of post-viral syndromes, also referred to as Myalgic Encephalomyelitis/Chronic Fatigue Syndrome, among other monikers, have been reported for at least three decades in the literature, some of which have been ascribed to influenza including its pandemic form [81, 82]. The findings summarized here also highlight the substantial 
burden of cardiopulmonary and cardioembolic health loss that is possible in the medium- and long-term following acute influenza infection. Therefore, seasonal influenza vaccinations are well-positioned to potentially reduce chronic health loss following influenza infection through their cardioprotective effects.

Finally, given the "break" in epidemic influenza infections in 2020, there is uncertainty as to the severity of future influenza outbreaks on a global scale. Therefore, it is imperative to ameliorate and maintain robust rates of seasonal influenza vaccination along with surveillance, monitoring, and preparedness through the Global Influenza Surveillance and Response System and regional bodies. Developing a four-strain influenza vaccine with newer vaccine platform technologies, analogous to mRNA vaccines for COVID19 , will likely not be as easy $[83,84]$. Therefore, we must maximize leveraging current seasonal influenza vaccine technologies, spanning from egg to cell-based, recombinant, and adjuvants, that have been repeatedly demonstrated to deliver safe and effective protection in the short, medium, and long terms.

Funding Ms. Behrouzi has been supported in part by a University of Toronto MD/PhD studentship award, a CANHEART SPOR Graduate Studentship Award, and a Ted Rogers Centre for Heart Research Doctoral Award. Dr. Udell has been supported by an Ontario Ministry of Research, Innovation and Science Early Researcher Award, Women's College Research Institute and the Department of Medicine at Women's College Hospital, and a Department of Medicine Merit Award, University of Toronto.

\section{Compliance with Ethical Standards}

Conflict of Interest Dr. Udell has received grant support to his institutions from AstraZeneca, Novartis, and Sanofi; has served as a consultant for Amgen, Boehringer Ingelheim, Janssen, Merck, Novartis, and Sanofi; and has received honoraria from Boehringer Ingelheim and Janssen. All authors have reported that they otherwise have no other relationships relevant to the contents of this paper to disclose and have completed and submitted the ICMJE Form for Disclosure of Potential Conflicts of Interest. The analyses, conclusions, opinions, and statements expressed herein are solely those of the authors and do not reflect those of the funding or data sources. No endorsement is intended nor should be inferred. Reference to specific commercial products, manufacturers, companies, or trademarks does not constitute its endorsement or recommendation by the U.S. Government, Department of Health and Human Services, or Centers for Disease Control and Prevention and any material referenced here is otherwise available on the agency website for no charge.

Human and Animal Rights and Informed Consent All reported studies/ experiments with human or animal subjects performed by the authors have been previously published and complied with all applicable ethical standards (including the Helsinki declaration and its amendments, institutional/national research committee standards, and international/ national/institutional guidelines).

\section{References}

Papers of particular interest, published recently, have been highlighted as:

- Of importance

$\bullet$ Of major importance

1. Ahmad FB, Anderson RN. The leading causes of death in the US for 2020. JAMA. 2021. https://doi.org/10.1001/jama.2021. 5469.

2. Garber AM. Learning from excess pandemic deaths. JAMA. 2021;325:1729.

3. Clift AK, Coupland CAC, Keogh RH, et al. Living risk prediction algorithm (QCOVID) for risk of hospital admission and mortality from coronavirus 19 in adults: national derivation and validation cohort study. BMJ. 2020;371:m3731.

4. Udell JA, Behrouzi B, Sivaswamy A, et al. Clinical risk, sociodemographic factors, and SARS-CoV-2 infection over time in Ontario, Canada. medRxiv Prepr. 2021. https://doi.org/10.1101/ 2021.04.28.21256.

5. Walker TA, Waite B, Thompson MG, et al. Risk of severe influenza among adults with chronic medical conditions. J Infect Dis. 2020;221:183-90.

6. Cohen R, Babushkin F, Geller K, Finn T. Characteristics of hospitalized adult patients with laboratory documented Influenza A, $\mathrm{B}$ and respiratory syncytial virus - a single center retrospective observational study. PLoS One. 2019;14:e0214517.

7.• Chow EJ, Rolfes MA, O'Halloran A, et al. Acute cardiovascular events associated with influenza in hospitalized adults. Ann Intern Med. 2020;173:605-13 This is a cross-sectional study of FluSurv-NET to determine risk factors for acute $\mathrm{HF}$ and acute ischemic heart disease in adults with a hospitalization associated with laboratory-confirmed influenza.

8. Prasad N, Walker TA, Waite B, et al. Respiratory syncytial virus-associated hospitalizations among adults with chronic medical conditions. Clin Infect Dis. 2020. https://doi.org/10. 1093/cid/ciaa730.

9. Chuaychoo B, Ngamwongwan S, Kaewnaphan B, Athipanyasilp N, Horthongkham N, Kantakamalakul W, Muangman N. Clinical manifestations and outcomes of respiratory syncytial virus infection in adult hospitalized patients. J Clin Virol. 2019;117:103-8.

10. Ivey KS, Edwards KM, Talbot HK. Respiratory syncytial virus and associations with cardiovascular disease in adults. J Am Coll Cardiol. 2018;71:1574-83.

11. Madjid M, Safavi-Naeini P, Solomon SD, Vardeny O. Potential effects of coronaviruses on the cardiovascular system. JAMA Cardiol. 2020. https://doi.org/10.1001/jamacardio.2020.1286.

12. Behrouzi B, Araujo Campoverde MV, Liang K, et al. Influenza vaccination to reduce cardiovascular morbidity and mortality in patients with COVID-19. J Am Coll Cardiol. 2020;76:177794 This narrative review provides a thorough summary of landmark studies assessing the association between influenza and CVD, as well as the cardioprotective effects of influenza vaccine.

13. Kwong JC, Schwartz KL, Campitelli MA, et al. Acute myocardial infarction after laboratory-confirmed influenza infection. $\mathrm{N}$ Engl J Med. 2018;378:345-53.

14. Corrales-Medina VF, Madjid M, Musher DM. Role of acute infection in triggering acute coronary syndromes. Lancet Infect Dis. 2010;10:83-92.

15. Warren-Gash C, Smeeth L, Hayward AC. Influenza as a trigger for acute myocardial infarction or death from cardiovascular disease: a systematic review. Lancet Infect Dis. 2009;9:601-10. 
16. Smeeth L, Thomas SL, Hall AJ, Hubbard R, Farrington P, Vallance P. Risk of myocardial infarction and stroke after acute infection or vaccination. N Engl J Med. 2004;351:2611-8.

17. Collins S. Excess mortality from causes other than influenza and pneumonia during influenza epidemic. Public Health Rep. 1932;47:2159-79.

18. Thompson WW, Shay DK, Weintraub E, Brammer L, Cox N, Anderson LJ, Fukuda K. Mortality associated with influenza and respiratory syncytial virus in the United States. JAMA. 2003;289:179.

19. Thompson WW, Shay DK, Weintraub E, Brammer L, Bridges $\mathrm{CB}$, Cox NJ, Fukuda K. Influenza-associated hospitalizations in the United States. J Am Med Assoc. 2004;292:1333.

20. Centers for Diseases Control and Prevention (CDC). Flu \& people with heart disease or history of stroke. In: Who is High Risk Flu Complicat. 2021. https://www.cdc.gov/flu/highrisk/heart disease.htm. Accessed 29 Mar 2021.

21. Centers for Diseases Control and Prevention (CDC). Estimated influenza illnesses, medical visits, hospitalizations, and deaths in the United States - 2019-2020 influenza season. 2020. https://www.cdc.gov/flu/about/burden/2019-2020.html\#: : text $=\mathrm{CDC} \% 20$ estimates $\% 20$ that $\% 20$ the $\% 20$ burden,flu $\% 20 \mathrm{dea}$ ths (Table 1). Accessed 7 July 2021.

22. Iuliano AD, Roguski KM, Chang HH, et al. Estimates of global seasonal influenza-associated respiratory mortality: a modelling study. Lancet. 2018;391:1285-300.

23. Sellers SA, Hagan RS, Hayden FG, Fischer WA. The hidden burden of influenza: a review of the extra-pulmonary complications of influenza infection. Influenza Other Respi Viruses. 2017;11:372-93.

24. Garten R, Blanton L, Elal AIA, et al. Update: influenza activity in the United States during the 2017-18 season and composition of the 2018-19 influenza vaccine. MMWR Morb Mortal Wkly Rep. 2018;67:634-42.

25. Centers for Diseases Control and Prevention (CDC). Flu activity \& surveillance. 2020. https://www.cdc.gov/flu/weekly/fluactivit ysurv.htm. Accessed 7 July 2021.

26. Schattner A. Acute cardiovascular events associated with influenza in hospitalized adults. Ann Intern Med. 2021;174:583.

27. Ludwig A, Lucero-Obusan C, Schirmer P, Winston C, Holodniy M. Acute cardiac injury events $\leq 30$ days after laboratoryconfirmed influenza virus infection among U.S. veterans, 20102012. BMC Cardiovasc Disord. 2015;15:109.

28. Chang T-Y, Chao T-F, Liu C-J, Chen S-J, Chung F-P, Liao J-N, Tuan T-C, Chen T-J, Chen S-A. The association between influenza infection, vaccination, and atrial fibrillation: a nationwide case-control study. Hear Rhythm. 2016;13:1189-94.

29. Reed C, Chaves SS, Daily Kirley P, et al. Estimating influenza disease burden from population-based surveillance data in the United States. PLoS One. 2015;10:e0118369.

30. Schneider A, Panagiotakos D, Picciotto S, et al. Air temperature and inflammatory responses in myocardial infarction survivors. Epidemiology. 2008;19:391-400.

31. Bhaskaran K, Hajat S, Haines A, Herrett E, Wilkinson P, Smeeth L. Short term effects of temperature on risk of myocardial infarction in England and Wales: time series regression analysis of the Myocardial Ischaemia National Audit Project (MINAP) registry. BMJ. 2010;341:c3823-c3823.

32. Warren-Gash C, Bhaskaran K, Hayward A, Leung GM, Lo S-V, Wong C-M, Ellis J, Pebody R, Smeeth L, Cowling BJ. Circulating influenza virus, climatic factors, and acute myocardial infarction: a time series study in England and Wales and Hong Kong. J Infect Dis. 2011;203:1710-8.

33. Warren-Gash C, Blackburn R, Whitaker H, McMenamin J, Hayward AC. Laboratory-confirmed respiratory infections as triggers for acute myocardial infarction and stroke: a self-controlled case series analysis of national linked datasets from Scotland. Eur Respir J. 2018;51:1701794.

34. Blackburn R, Zhao H, Pebody R, Hayward A, Warren-Gash C. Laboratory-confirmed respiratory infections as predictors of hospital admission for myocardial infarction and stroke: timeseries analysis of English data for 2004-2015. Clin Infect Dis. 2018;67:8-17.

35. Kytömaa S, Hegde S, Claggett B, Udell JA, Rosamond W, Temte J, Nichol K, Wright JD, Solomon SD, Vardeny O. Association of influenza-like illness activity with hospitalizations for heart failure. JAMA Cardiol. 2019;4:363.

36. Ohland J, Warren-Gash C, Blackburn R, Mølbak K, Valentiner-Branth P, Nielsen J, Emborg H-D. Acute myocardial infarctions and stroke triggered by laboratory-confirmed respiratory infections in Denmark, 2010 to 2016. Eurosurveillance. 2020. https://doi.org/10.2807/1560-7917.ES.2020.25. 17.1900199.

37. García-Lledó A, Rodríguez-Martín S, Tobías A, García-de-Santiago E, Ordobás-Gavín M, Ansede-Cascudo JC, Alonso-Martín J, de Abajo FJ. Relationship between influenza, temperature, and type 1 myocardial infarction: An ecological time-series study. J Am Heart Assoc. 2021;10:e019608.

38. Udell JA, Zawi R, Bhatt DL, et al. Association between influenza vaccination and cardiovascular outcomes in high-risk patients. JAMA. 2013;310:1711.

39. Clar C, Oseni Z, Flowers N, Keshtkar-Jahromi M, Rees K. Influenza vaccines for preventing cardiovascular disease. Cochrane Database Syst Rev. 2015. https://doi.org/10.1002/14651858. CD005050.pub3.

40. Rodrigues BS, Alves M, Duarte GS, Costa J, Pinto FJ, Caldeira $D$. The impact of influenza vaccination in patients with cardiovascular disease: an overview of systematic reviews. Trends Cardiovasc Med. 2020. https://doi.org/10.1016/j.tcm.2020.06. 003.

41. Yedlapati SH, Khan SU, Talluri S, et al. Effects of influenza vaccine on mortality and cardiovascular outcomes in patients with cardiovascular disease: a systematic review and meta-analysis. J Am Heart Assoc. 2021. https://doi.org/10.1161/JAHA.120. 019636.

42. Modin D, Jørgensen ME, Gislason G, Jensen JS, Køber L, Claggett B, Hegde SM, Solomon SD, Torp-Pedersen C, BieringSørensen T. Influenza vaccine in heart failure: cumulative number of vaccinations, frequency, timing, and survival: a Danish nationwide cohort study. Circulation. 2019. https://doi.org/10. 1161/CIRCULATIONAHA.118.036788.

43. Flather MD, Yusuf S, Køber L, et al. Long-term ACE-inhibitor therapy in patients with heart failure or left-ventricular dysfunction: a systematic overview of data from individual patients. Lancet. 2000;355:1575-81.

44. McAlister FA, Wiebe N, Ezekowitz JA, Leung AA, Armstrong PW. Meta-analysis: $\beta$-blocker dose, heart rate reduction, and death in patients with heart failure. Ann Intern Med. 2009;150:784.

45. Yancy CW, Jessup M, Bozkurt B, et al. 2017 ACC/AHA/HFSA focused update of the 2013 ACCF/AHA guideline for the management of heart failure: a report of the American College of Cardiology/American Heart Association Task Force on Clinical Practice Guidelines and the Heart Failure Society of Amer. Circulation. 2017. https://doi.org/10.1161/CIR.0000000000000509.

46. Behrouzi B, Udell JA. Influenza vaccine as part of a heart disease armamentarium in the new cardio-respiratory virus era. Trends Cardiovasc Med. 2020. https://doi.org/10.1016/j.tcm.2020.07. 001.

47. Fulop T, Larbi A, Dupuis G, Le Page A, Frost EH, Cohen AA, Witkowski JM, Franceschi C. Immunosenescence and 
inflamm-aging as two sides of the same coin: friends or foes? Front Immunol. 2018. https://doi.org/10.3389/fimmu.2017. 01960.

48. Franceschi C, Garagnani P, Parini P, Giuliani C, Santoro A. Inflammaging: a new immune-metabolic viewpoint for agerelated diseases. Nat Rev Endocrinol. 2018;14:576-90.

49. Vardeny O, Sweitzer NK, Detry MA, Moran JM, Johnson MR, Hayney MS. Decreased immune responses to influenza vaccination in patients with heart failure. J Card Fail. 2009;15:368-73.

50. Wu NC, Zost SJ, Thompson AJ, Oyen D, Nycholat CM, McBride R, Paulson JC, Hensley SE, Wilson IA. A structural explanation for the low effectiveness of the seasonal influenza H3N2 vaccine. PLOS Pathog. 2017;13:e1006682.

51. Paules CI, Sullivan SG, Subbarao K, Fauci AS. Chasing seasonal influenza - the need for a universal influenza vaccine. N Engl $\mathrm{J}$ Med. 2018;378:7-9.

52. Zost SJ, Parkhouse K, Gumina ME, Kim K, Diaz Perez S, Wilson PC, Treanor JJ, Sant AJ, Cobey S, Hensley SE. Contemporary $\mathrm{H} 3 \mathrm{~N} 2$ influenza viruses have a glycosylation site that alters binding of antibodies elicited by egg-adapted vaccine strains. Proc Natl Acad Sci. 2017;114:12578-83.

53. Nachbagauer R, Feser J, Naficy A, et al. A chimeric hemagglutinin-based universal influenza virus vaccine approach induces broad and long-lasting immunity in a randomized, placebocontrolled phase I trial. Nat Med. 2021;27:106-14.

54. Krammer F, Palese P. Advances in the development of influenza virus vaccines. Nat Rev Drug Discov. 2015;14:167-82.

55. DiazGranados CA, Dunning AJ, Kimmel M, et al. Efficacy of high-dose versus standard-dose influenza vaccine in older adults. N Engl J Med. 2014;371:635-45.

56. Van Ermen A, Hermanson MP, Moran JM, Sweitzer NK, Johnson MR, Vardeny O. Double dose vs. standard dose influenza vaccination in patients with heart failure: a pilot study. Eur $\mathrm{J}$ Heart Fail. 2013;15:560-4.

57. DiazGranados CA, Robertson CA, Talbot HK, Landolfi V, Dunning AJ, Greenberg DP. Prevention of serious events in adults 65 years of age or older: a comparison between highdose and standard-dose inactivated influenza vaccines. Vaccine. 2015;33:4988-93.

58. Vardeny O, Udell JA, Joseph J, et al. High-dose influenza vaccine to reduce clinical outcomes in high-risk cardiovascular patients: rationale and design of the INVESTED trial. Am Heart J. 2018;202:97-103.

59. Bhatt AS, Vardeny O, Udell JA, Joseph J, Kim K, Solomon SD. Influenza vaccination: a 'shot' at INVESTing in cardiovascular health. Eur Heart J. 2021. https://doi.org/10.1093/eurheartj/ ehab133.

$60 . \bullet$ Vardeny O, Kim K, Udell JA, et al. Effect of high-dose trivalent vs standard-dose quadrivalent influenza vaccine on mortality or cardiopulmonary hospitalization in patients with high-risk cardiovascular disease. JAMA. 2021;325:39 These are the recent results of the first large influenza vaccine cardiovascular outcome trial, INVESTED, in patients with high-risk CVD, including previous MI and HF hospitalizations.

61.• Hollingsworth R, Palmu A, Pepin S, Dupuy M, Shrestha A, Jokinen J, Syrjänen R, Nealon J, Samson S, De Bruijn I. Effectiveness of the quadrivalent high-dose influenza vaccine for prevention of cardiovascular and respiratory events in people aged 65 years and above: rationale and design of a real-world pragmatic randomized clinical trial. Am Heart J. 2021. https://doi.org/10. 1016/j.ahj.2021.03.007 This is the design of the newest large influenza vaccine cardiovascular outcome (registry-based) trial, conducted by the Finnish Institute for Health and Welfare, in patients aged 65 and up.
62.• Fröbert O, Götberg M, Angerås $\mathrm{O}$, et al. Design and rationale for the influenza vaccination After Myocardial Infarction (IAMI) trial. A registry-based randomized clinical trial. Am Heart J. 2017;189:94-102 This is the design of the first registry-based, large influenza vaccine cardiovascular outcome trial in patients with acute coronary syndrome undergoing coronary angiography.

63. Loeb M, Dokainish H, Dans A, et al. Randomized controlled trial of influenza vaccine in patients with heart failure to reduce adverse vascular events (IVVE): rationale and design. Am Heart J. 2019;212:36-44 This is the design of a large influenza vaccine cardiovascular outcome trial in patients with $\mathrm{HF}$.

64. Grohskopf LA, Alyanak E, Broder KR, Blanton LH, Fry AM, Jernigan DB, Atmar RL. Prevention and control of seasonal influenza with vaccines: recommendations of the advisory committee on immunization practices - United States, 2020-21 Influenza Season. MMWR Recomm Reports. 2020;69:1-24.

65. Nylén G, Linde A, Kettis AA, Wik O, Stenmark S, Erntell M. Health personnel the key to high vaccination coverage against influenza. Lakartidningen. 109:8-9

66. Ferrucci L, Fabbri E. Inflammageing: chronic inflammation in ageing, cardiovascular disease, and frailty. Nat Rev Cardiol. 2018;15:505-22.

67. Laurie KL, Rockman S. Which influenza viruses will emerge following the SARS-CoV-2 pandemic? Influenza Other Respi Viruses. 2021;15:573-576.

68. Young B, Zhao X, Cook AR, Parry CM, Wilder-Smith A, I-Cheng MC. Do antibody responses to the influenza vaccine persist year-round in the elderly? A systematic review and metaanalysis. Vaccine. 2017;35:212-21.

69. Myhre PL, Grundvold I, Paulsen TH, Omland T, Schirmer H. Cardiovascular hospitalizations, influenza activity, and COVID19 measures. Circulation. 2020;142:1302-4.

70. O'Halloran AC, Lu P, Williams WW, Bridges CB, Singleton JA. Influenza vaccination coverage among people with high-risk conditions in the U.S. Am J Prev Med. 2016;50:e15-26.

71. Grohskopf LA, Alyanak E, Broder KR, Walter EB, Fry AM, Jernigan DB. Prevention and control of seasonal influenza with vaccines: recommendations of the advisory committee on immunization practices - United States, 2019-20 Influenza Season. MMWR Recomm Rep. 2019;68:1-21.

72. Smith SC, Allen J, Blair SN, et al. AHA/ACC guidelines for secondary prevention for patients with coronary and other atherosclerotic vascular disease: 2006 Update. Circulation. 2006;113:2363-72.

73. Grandhi GR, Mszar R, Vahidy F, Valero-Elizondo J, Blankstein R, Blaha MJ, Virani SS, Andrieni JD, Omer SB, Nasir K. Sociodemographic disparities in influenza vaccination among adults with atherosclerotic cardiovascular disease in the United States. JAMA Cardiol. 2020. https://doi.org/10.1001/jamacardio.2020. 3978.

74. Sperling LS, Albert MA, Koppaka R. Disparities in influenza vaccination-opportunity to extend cardiovascular prevention to millions of hearts. JAMA Cardiol. 2020. https://doi.org/10. 1001/jamacardio.2020.3983.

75. WHO (World Health Organization). Ten threats to global health in 2019. 2019. https://www.who.int/news-room/spotlight/tenthreats-to-global-health-in-2019. Accessed 7 July 2021.

76. Lazarus JV, Ratzan SC, Palayew A, Gostin LO, Larson HJ, Rabin K, Kimball S, El-Mohandes A. A global survey of potential acceptance of a COVID-19 vaccine. Nat Med. 2021;27:225-8.

77. WHO (World Health Organization). Report of the SAGE working group on vaccine hesitancy. 2014. https://www.who.int/ immunization/sage/meetings/2014/october/1_Report_WORKI NG_GROUP_vaccine_hesitancy_final.pdf. Accessed 7 July 2021. 
78. Schattner A. Cardiovascular-targeted patient education and uptake of influenza vaccination in elderly patients. Patient Educ Couns. 2020;103:1052-4.

79. Bhugra P, Grandhi GR, Mszar R, Satish P, Singh R, Blaha M, Blankstein R, Virani SS, Cainzos-Achirica M, Nasir K. Determinants of influenza vaccine uptake in patients with cardiovascular disease and strategies for improvement. J Am Heart Assoc. 2021;10:e019671.

80. Al-Aly Z, Xie Y, Bowe B. High-dimensional characterization of post-acute sequelae of COVID-19. Nature. 2021. https://doi.org/ 10.1038/s41586-021-03553-9.

81. Proal A, Marshall T. Myalgic encephalomyelitis/chronic fatigue syndrome in the era of the human microbiome: persistent pathogens drive chronic symptoms by interfering with host metabolism, gene expression, and immunity. Front Pediatr. 2018. https:// doi.org/10.3389/fped.2018.00373.
82. Magnus P, Gunnes N, Tveito K, Bakken IJ, Ghaderi S, Stoltenberg C, Hornig M, Lipkin WI, Trogstad L, Håberg SE. Chronic fatigue syndrome/myalgic encephalomyelitis (CFS/ME) is associated with pandemic influenza infection, but not with an adjuvanted pandemic influenza vaccine. Vaccine. 2015;33:6173-7.

83. Abbasi J. The search for a universal flu vaccine heats up. JAMA. 2019;322:1942.

84. Eisenstein M. Towards a universal flu vaccine. Nature. 2019;573:S50-2.

Publisher's Note Springer Nature remains neutral with regard to jurisdictional claims in published maps and institutional affiliations. 